Journal of Computer Science and Technology Studies (JCSTS))

ISSN: $2709-104 \mathrm{X}$

DOI: $10.32996 / j$ csts

Journal Homepage: www.al-kindipublisher.com/index.php/jcsts

\title{
Electronic Document Management System (EDMS) Implementation: Implications for the Future of Digital Transformation in Philippine Healthcare
}

\author{
Alyssa Marie B. Almacen ${ }^{1} \square$ and Alan Y. Cabaluna, Ph.D ${ }^{2}$ \\ ${ }^{1}$ La Consolacion University Philippines, Malolos, Philippines \\ ${ }^{2}$ Don Honorio Ventura State University, Candaba Extension Campus, Philippines
}

$\square$ Corresponding Author: Alyssa Marie B. Almacen, E-mail: alymarie.almacen@gmail.com

\section{ARTICLE INFORMATION ABSTRACT}

Received: 04 October 2021

Accepted: 21 November 2021

Published: 22 December 2021

DOI: $10.32996 /$ jcsts.2021.3.2.8

\section{KEYWORDS}

EDMS implementation, Electronic Document Management Systems, Healthcare, Records, Documentation, Future, Effects, Record-Keeping
The COVID-19 pandemic is raising concerns all around the globe, as many lives have been lost and families have been left without hope. The Digital Era has started, and many sectors, including healthcare, are adapting to the innovative human-machine integration that has developed. However, the new systems still offer benefits and limits that will influence certain nations' choice to accept this platform owing to their unique requirements and circumstances. The earliest electronic health records were developed in the early 1960s to store long-term patient data. However, it was not maintained since the system's foundation is very demanding, and medical practitioners stopped utilizing it. After a decade, the growth of technology was unavoidable, gradually altering areas such as business and healthcare. However, every development has ramifications. The implementation of the new system may enhance organizational performance, but there are still uncertainties, notably about sustainability and how it will affect the firm in the future. Now, sufficient medical documentation has long been a source of contention in the healthcare industry, and management should begin introducing new processes to address the underlying problem. If the government already has a system in place to ensure the country's long-term existence, this system should be used to ensure the consistency of each patient's medical records. Will an Electronic Document Management System (EDMS) adaptation in the Philippines, a third-world nation with a poor healthcare system, be the best option to modify and enhance medical care quality?

\section{Introduction}

The medical record has typically been handwritten using pen and paper, and this technique has stood the test of time. In contrast, regardless of how secure the filing storage is, paper degrades, and pen-written files fade with time, endangering the long-term viability of record-keeping. Consistency in documentation is highly valued in the healthcare sector since it allows for monitoring patients' health, which may prevent larger concerns from happening later in their lives. Furthermore, fire, insects, and even water can entirely destroy paper archives. Computerized access to patients' medical records has been proved useful in first-world countries for many years. According to N. Kain and O. Koshy, patient records in the United Kingdom, for example, have increased due to an unsustainable demographic scenario dominated by people over the age of 60 . As a result, the quantity of files has risen considerably. Because of a lack of space and the related high cost of safe-keeping facilities, their record-keeping has become more difficult throughout their lifetime. Furthermore, since patient care is distributed across medical institutions, record transfer has proven to be time-consuming and costly. These incidents occurred before the use of EDMS in their healthcare delivery system. In the United Kingdom, electronic document management systems (EDMS) are now referred to as digital healthcare solutions.

Copyright: (C) 2021 the Author(s). This article is an open access article distributed under the terms and conditions of the Creative Commons Attribution (CC-BY) 4.0 license (https://creativecommons.org/licenses/by/4.0/). Published by Al-Kindi Centre for Research and Development, London, United Kingdom. 


\section{Literature Review}

\subsection{The Benefits and Drawbacks of Paper Patient Record}

There was no systematic recording of the advantages of paper patient records in the healthcare industry until recently. Part of the reason for this outcome may be due to widespread recognition in the medical profession of the need to maintain patient records and the fact that paper is commonly utilized in maintaining records. Considering the ubiquitous use of paper medical records, the healthcare industry has emphasized the importance of practitioners' cooperation for this type of record-keeping. Although healthcare industries were unable to investigate user viewpoints on paper records due to time and resource restrictions, they were able to identify five of the advantages of paper records from the standpoint of firsthand users:

1. Because record-keeping in the paper is known to users, they have not required the acquisition of new skills or the development of new habits to utilize them.

2. The paper record is easily transportable and may be brought to the location of treatment with the patient.

3. In contrast to computer systems, paper records do not need any upkeep once they are in your hands.

4. Documentation in the paper gives greater versatility in collecting data and is more likely to capture "soft" information.

5. It is possible to see and scan paper records. Users may use this component to outline the data in a number of strategies and look for a basis that isn't explicitly addressed elsewhere in the program, among other things.

Patient's current medical records are often criticized. Specifically, according to Burnum (1989), "long faulty patient records today include more modified, erased, and false material than at any time in history." According to Pories, an engineer was tasked with advocating for more effective use of healthcare professionals but instead was "stunned by the chaos of the medical record and the inefficiencies it imposed on the delivery of treatment" (1990). According to the engineer, "the record layout gave the most obvious and straightforward strategy for medical expense reduction and responsibility avoidance".

In his statement, Pories claims that the situation has not improved and that this is not a unique incident in the nation. According to the evidence, the problem does not seem to be restricted to a single region: health records seem to be equally inaccurate and problematic across the globe (Pories 1990). Apparently, he is not alone in believing that patient data often lack the elements necessary for making the most of the data contained inside them. An internist has discovered in the institution and private practice that patients' records are becoming more demanding, with 63 percent of those who participated in the survey believing that this is happening despite an improvement in the quality of patient treatment (Hershey et al., 1989).

According to the literature and the committee's work, patients' records have several shortcomings, which can be classified into four categories: content, format, linkages, and integration.

\subsection{The Emergence of EDMS in Healthcare}

The process of delivering high-quality healthcare services is mostly driven by data collection and analysis. Information processing skills, such as how effectively data is digested, restored, and introduced, have even been characterized as "dominating" medical practice (Barnett, 1990). According to estimates, approximately 35 to 39 percent of overall hospital's operating costs are attributed to patient and professional communication initiatives (Richart, 1970). Approximately 38 percent of physicians' time (Mamlin and Baker, 1973) and almost 50 percent of nurses' time (Korpman and Lincoln, 1988) is spent inputting data into patients' medical records.

The information-processing methods utilized to deliver healthcare services to patients are quite diverse. Patient information is collected and recorded by clinicians, who then consult with colleagues and read scientific literature before selecting diagnostic procedures, interpreting laboratory results, developing patient care strategies, instructing allied health professionals, discussing care plans with patients and families, and documenting patients' progress. Medical decision-makers must also be able to condense and assess information while also using their understanding and navigating the difficulties of decision-making (Haynes et al., 1989; Greenes and Shortliffe, 1990). As a result, to make prompt and educated choices about patient treatment, health care practitioners often require access to relevant compilations of comprehensive latest information and recommendations (Haynes et al., 1989; Saba et al., 1989; Greenes and Shortliffe, 1990). A broad range of information-processing tasks is used to support patient care, including the performance of laboratory tests, the analysis of medical imaging data, the collection of patient information, categorizing of prescription orders, the tracking and assessment of the quality and proper services provided, and the charging of services (Martin, 1990).

\subsection{Challenges of EDMS: Increasing Data Demand}

Although patient care data is getting increasingly extensive and complicated, there is an increasing desire for many individuals to access information about patient care (Barnett, 1990). The term "health care team" refers to a varied set of healthcare professionals who must collaborate in order to deliver appropriate treatment to patients. The following occupations fall within this category: 
physicians, nurses, therapists, medical technicians, dentists, pharmacists, social workers, and other members of the healthcare professions. Patients may also express a desire to have access to their medical records. In order to increase patient participation, some providers recommend that patients identify their treatment preferences, contribute to the record (particularly history and functional status; Davies and Ware, 1988; Tarlov and colleagues, 1989), read and validate the record data, control the record and pertinent transport parts of the record to the provider's office or facility (Tufo et al. 1977; Bronson et al., 1978).

Administrators and managers of health care facilities depend on information to monitor the quality of treatment offered and allocate resources (such as staff, supplies, equipment, and buildings) according to the institution's patient case mix. In order to develop realistic budgets, analyze productivity and expenses, and estimate their market position, managers of provider institutions strive to combine financial data with patient care data in order to develop realistic budgets, analyze productivity and expenses, and estimate their market position. Changes in patient population demand are predicted to impact the long-term planning of the institution, including personnel recruitment, equipment purchase, and facility expansion plans.

\subsection{Challenges of EDMS: Maintaining Confidentiality}

As a result of these changes, the increasing availability and demand for medical data have created a critical need for patient privacy protection, which must be addressed swiftly to avoid further delay. Healthcare workers are bound by an old idea of Confidentiality, which compels them to avoid infringing on a patient's right to privacy in the course of their work. One of the most difficult challenges in designing future health records systems will be striking a reasonable balance between patient Confidentiality and the capacity of users to obtain information when they have a legitimate reason to do so.

\subsection{Benefits of EDMS}

A commonly held belief is that electronic document management systems (EDMS) are one of the most important development strategies in digital healthcare innovation, and their widespread adoption in industrialized nations has had a significant influence on the field's advancement. For example, the government of the United Arab Emirates, specifically the emirate of Abu Dhabi, has successfully implemented a system called Malaffi, which is an Arabic term that translates as "my file," to promote health informatics and eHealth as part of the country's digital transformation of its healthcare system as part of the country's digital transformation of its healthcare system. The system is anticipated to increase patient experience and happiness while also providing patients and medical practitioners with the confidence that they are getting and delivering the finest, safest, and most timely medical treatment possible.

Using a traditional clinical record system has several drawbacks that outweigh the benefits of using an electronic medical record system (such as paper records). Long overdue, particularly in the Philippines, where the government has been unable to keep up with the demand for such services, is the establishment of an integrated database of actual public health information at one location to aid in epidemic prevention, as well as the development of rapid response action plans, are created to protect the public's health and well-being. It is difficult for the doctor who is scheduled to meet with the patient to access the patient's information on the computer because of the enormous number of outpatient clinic appointments booked on consecutive days. Because of the challenges in accessing the most critical medical information, physicians are often frustrated, which may harm patient treatment outcomes. Patient records may be difficult to retrieve if they are not immediately available when needed. This is especially true if the patient is illiterate, drunk, or has learning impairments, as is the case in many cases. Getting access to records will likely be difficult if the records are not instantly accessible when requested. Every interaction with a medical professional, whether it is a regular checkup, doctor's appointment, or emergency medical care, and reports from laboratories and radiology, is collected in a single patient record, ensuring that their medical background is stored securely is easily accessible when needed. EDMS will continue to play a significant role in the community for the foreseeable future.

\subsection{The Need of EDMS in Healthcare}

Numerous software was being developed through the years to create medical software, restructure the patient file, and promote medical practitioners to preserve their records more logically and systematically by entering necessary clinical data).

According to research, there are five external circumstances wherein the organization's overall strategic plan could be executed in order to maximize the chance of widespread adoption of digital medical records. These situations are: The current need for patient information in the healthcare business is unlikely to reduce in the foreseeable future, and it is predicted to become even more so in the near future. For the second time, paperless patient record systems continue to improve in terms of their utility and cost, and it is projected that this trend will continue. A third factor to consider is that patients and practitioners are becoming more used to employing technology in almost every part of their daily lives, which is a trend that is expected to continue. For the fourth time, an ageing and moving population will entail more data management and a greater demand for enhanced information transferability or portability, among other things. Finally, without modifications to the scope, usage, and automation of the patient 
record, it would be impossible to accomplish the critical components of healthcare reform that need evaluation, data consolidation, and increased communication, to name a few requirements.

The significance and role of the Electronic Document Management System (EDMS) in providing immediate access to critical patient health data, improving patient shift of care and care collaboration, reducing excessive usage and redundancy of diagnostics, diagnostic imaging examinations, and other screening tests, and enabling the practice of precision medicine will be better understood and appreciated as a result of its implementation in Philippine healthcare. Among other things, the objective of this research is to dive further into the application and use of document management in terms of data security, system sustainability, and long-term record-keeping.

This Study will benefit the Department of Health, the healthcare industry, medical practitioners, and patients by enabling them to keep an eye on medical data that may be beneficial in the future diagnosis of their issues to prevent them from becoming worse.

\subsection{Research Framework and Hypotheses Development}

Electronic medical record systems (EDMS) must integrate all necessary information in their role in the healthcare system. Given the legal and financial capacities of the healthcare business, records must be accurate and complete. According to expectations, paper papers will continue to be a significant element of a valid medical record for the following years (Stratis Health, 2009).

The Philippines is one of the few countries where the Electronic Document Management System (EDMS) is not commonly utilized in healthcare. Only companies with enough financial resources seem to invest in a paperless system. The need for a national EDMS has risen rapidly due to fragmented and inefficient patient care delivery and inconsistency in diagnosis due to the loss of medical history information. A complete evaluation of the EDMS system as a healthcare system is required to understand industry expectations and find solutions to present challenges.

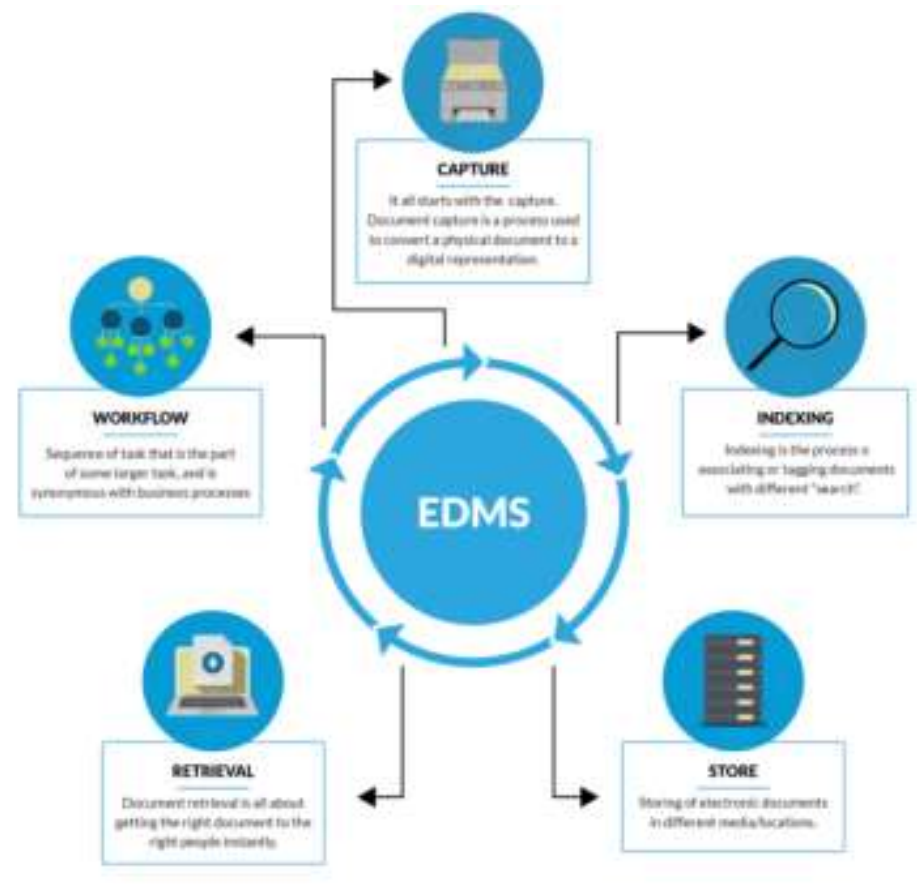

Figure 1. EDMS Mechanism

Figure 1 displays several Philippines' available technologies to help you get started with system integration and full document automation, ranging from storage to basic paper digitalization.

a. In a hierarchical file, digital documents are gathered into a central store based on envelope structures, which contain reports, which are then classified by the office, date, or other data administration attributes.

b. Data is retrieved from record-keeping structures such as other databases, based on the document management software system-wide, in order to naturally capture, track, and connect to data received during the project. 
c. Finding records in various archive envelopes and warehouses using federated search - EDMS enables authorized representatives to retrieve documents from various archive envelopes and warehouses by using search options that typically include the document name, report content, and other information.

d. Records are established for each representative job or division, and data is provided to the relevant individuals.

e. Security and audit trails are also accessible. As a result, audit trails may be utilized to show precisely how data is accessed, ensuring more administrative consistency.

\subsection{The Fundamentals of Choosing an EDMS}

Developing a more thorough approach should begin with the fundamental steps in Figure 1 that outline the process of selecting, implementing, and operating an enterprise content management system. As a general rule, the following is the method to follow

- Requirements gathering and analysis. First and foremost, you must determine your own requirements. Before implementing an EDMS, ensure that the long-term criteria for reliability, completeness, accessibility, legal admissibility and durability are acknowledged.

- The selection of vendors. The selection of an appropriate EDMS service provider. To solicit bids, it is required to submit a request for bids that clearly outlines both the legal requirements and the selection criteria to be used when selecting bidders. Make a plan for putting it into action. It is vital to put together a comprehensive implementation strategy. The following elements should be included in the strategy:

- It is necessary to develop a technology implementation strategy that details the method and timing of the system's deployment and testing.

- User implementation tactics involve user training and system deployment, amongst other activities.

- Deployment. System validation, installation, and training of users are all required as part of the implementation process.

- Management. While the system is in operation, it is critical to retain control over and enhance its usage.

It is critical to record the whole process at each phase, including the requirements assessment, implementation, management, and refining. System records must be documented throughout the system's life to guarantee that they stay dependable throughout time. This includes documentation of the hardware, software, operating processes, and security methods.

\subsection{Factors in Implementing EDMS in the Philippines}

\subsection{Economic Situation - Huge Investment}

One of the factors to consider while implementing EDMS in the Philippines is the value for money. Because the system must meet specific specifications in order to meet the needs of a single healthcare system, significant funds must be spent to provide what the healthcare system requires, especially in medical billing, patient record-keeping, and the "paperless-based system," which is accessible to both medical practitioners and patients at any time and from any location.

The country's healthcare investment strategy is lacklustre, and as a result, we are one of the world's worst providers of patient care. A lack of funding for medical facilities and practitioners and the risk of not investing in this kind of system are all considerations. One of the most serious flaws in the Philippine healthcare system is failing to keep accurate patient records and monitor medical history. Investing in a system that can automate medical information retrieval, tracking, and storage, among other functions, is a low-cost solution to solve these concerns.

\subsubsection{The big switch to a "paperless" system.}

The majority of localities in the Philippines have minimal or no access to digital solutions. Because of the inequitable access, the available solutions are scattered. Physical impediments, such as sluggish internet, are not the sole causes for change. Compared to other nations, the Philippines is slowly adopting technology; hence there are certain misunderstandings about it. For medical practitioners and patients alike, the conventional "paper-based" technique is time-consuming and labour-intensive, and it has multiple fundamental disadvantages. Historically, medical practitioners and administrative workers communicated primarily via paper forms and faxes. Exchanging information is time-consuming and difficult since paper forms are lost, paper notes must be physically transported or faxed, notes may be illegible, and pages are missing. The transition to the paperless system may be a big problem in the Philippines; however, through EDMS, costs and time are decreased, and more information is accessible.

\subsubsection{Efficacy in the Clinical Setting}

Despite being extremely difficult in practice in the Philippines, it can be implemented through randomized clinical practices, allowing for improvements in population health outcomes as well as the use of adopted outcome measures that focus on the quality and coordination of health care, as well as disease surveillance in the context of population health management. 
Few studies show that some healthcare institutions in developed countries have already digitalized their basic administrative processes, such as patient admission, centralized consultations, discharge, or medical episode recording with relevant coding. This is attributed to the reason that information is sent promptly, reducing the time between requests. As a consequence, statistically significant outcomes, such as lower death rates and after treatment expenses for acute diseases, as well as increased profits in the services supplied, are anticipated.

Patient care may be within people' access in an archipelagic state like the Philippines, and the underserved will be addressed faster since information is accessible at all times. Paper records are time-consuming and labour-intensive to gather, and employees must spend substantial time identifying and filing the necessary materials in the correct sequence. A number of complaints have been filed, most of which have been documented, involving delays in sending information to patients, miscommunications, missing information, and massive amounts of paper waste generated as a result of the paper-based system. Records have grown increasingly difficult to maintain since staff members working in healthcare facilities do not have the time or resources to obtain the documentation they need to execute their jobs effectively.

Using an EDMS will help employees work more efficiently while also boosting their safety. Patients will gain a lot because practitioners will be able to continue to give the high-quality care to which they have been accustomed. Improved data from various sources and departments, which will be available sooner rather than later, will improve decision-making in the clinic's working environment. Because medical practitioners will have more time to prepare and communicate with patients transparently due to quick information sharing, faster information interchange among disciplines will result in more tailored treatment.

\subsubsection{Patient Satisfaction}

The amount of patient satisfaction is determined by how patients were handled at the hospital. Dissatisfaction may emerge due to the repetitive transmission of information to a large number of medical practitioners, the delayed reporting of medical information, or the delivery of redundant or unnecessary tests and treatments.

According to Doyle, C. et al. (2013), patient experience is positively related to clinical efficacy and patient safety, supporting the argument for patient experience to be included as one of the key pillars of quality in healthcare. An evaluation of interventions to improve medication adherence and patient care identified informative communication, excellent provider-patient relationships, and patients' agreement with the necessity for treatment as common predictors of effectiveness.

Paperless records will provide the most up-to-date and accurate information, enabling faster diagnosis, treatment plans, and patient discharge processes. By providing access to complete, up-to-date medical information, we can improve patient safety and satisfaction with the healthcare system while reducing the chance of clinical error. If the Philippine healthcare system can enhance clinical efficiency in at least common clinical procedures, it would significantly influence patient satisfaction. 


\section{Methodology}

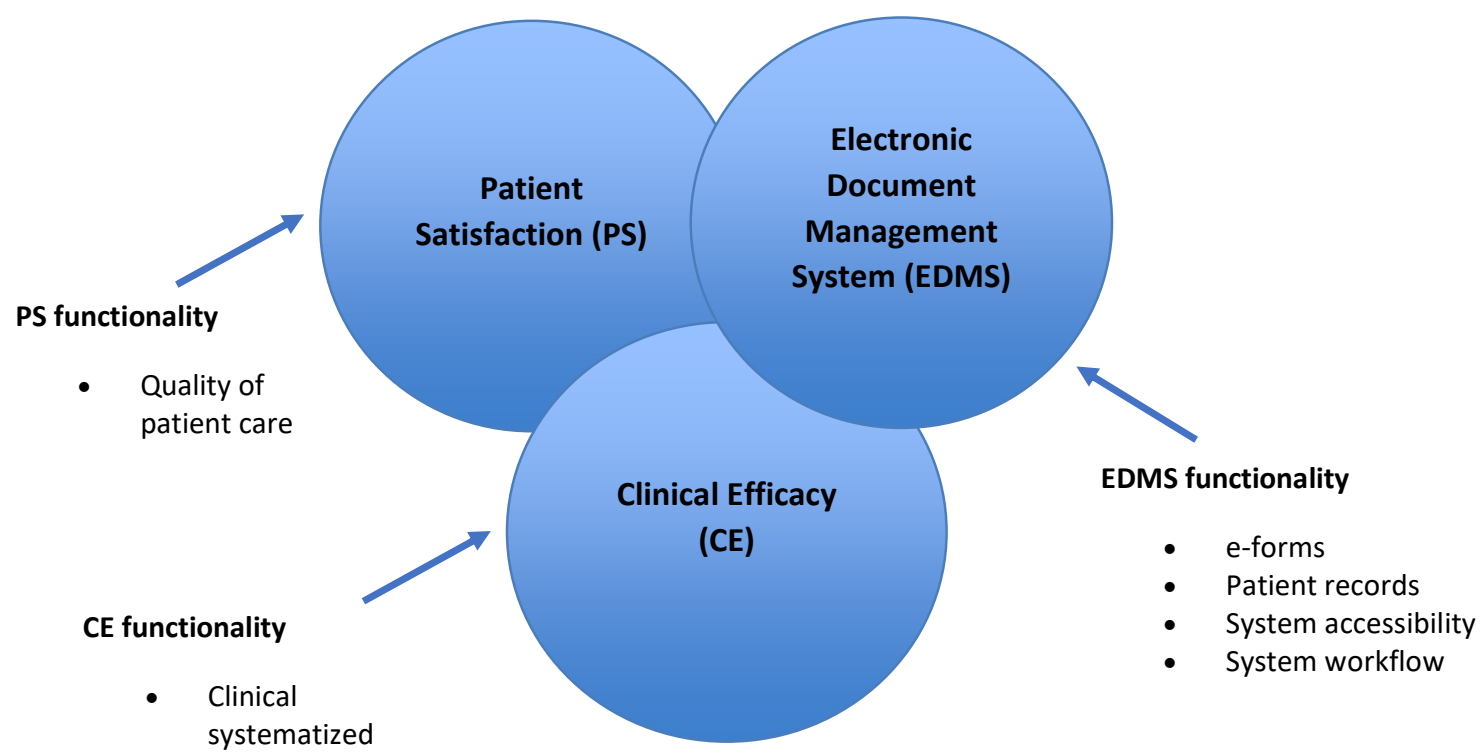

operation

Figure 2. The framework of the Study

This diagram depicts the groundwork for empirical studies on the correlation of the variables. The research framework explains how the identified variables are related to one another. As the major workflow driver, the EDMS has a considerable impact on clinical effectiveness and patient satisfaction. Adoption of EDMS, which may include e-forms and expedited patient records, system accessibility, and well-organized workflow, increases patient satisfaction and a feeling of being cared for. This leads to a more systematized clinical operation (for example, smooth visits, accurate treatment plans, and diagnosis), which improves patient care quality and promotes patient satisfaction and emotions of well-being.

\subsection{Participants of the Study}

Participants in the Study were selected through various methods, including secondary data collection and observation. Because the study topic had not yet been fully implemented in Philippine healthcare settings, it was decided to collect data from other published sources to augment the research data that would be used in any future implementation of the research findings. The observational approach, on the other hand, must be used as part of the World Health Organization's "systematic public inquiry" (Stenhouse, 1975). The observation entails more than simply seeing and hearing, and it is therefore selective in certain ways. To undertake systematic observation, the research team must first determine what they will be searching for. To appraise the situation as naturally as possible, the information that has been acquired must be examined and processed utilizing all of the senses. Patients in the Philippines were limited to locals and included medical practitioners and patients from various healthcare professions spread over the archipelago's 7,000 islands.

\subsection{Research Instruments}

A variety of research techniques were used in the investigation. The various research approaches were categorized into two (2) groups based on the various research approaches. The first section's content was gathered from publicly accessible, both published and unpublished, websites. Unpublished resources include firsthand research, opinions, and points of view expressed by random individuals and organizations; on the other hand, published resources include international and domestic government publications, healthcare policies and protocols, business cases, and technical/trade journals. The observation method was employed to gather the instruments' second component, which could be classified as organized or unstructured. Data was gathered using certain variables and on a set timetable via organized or systematic observation. There were no predefined ideas or goals to consider; therefore, an open and free approach was adopted using unstructured observation.

This Study's results may be better understood using observation as à technique since it can be used in 'natural' scenarios rather than ones that are intentionally set up for research goals, such as interviews. In order to get a better understanding of social processes and occurrences, the application of observation to these paradigms may be useful. The approach included creating a typology of dying 'consciousness settings' based on observational data, illustrating the process involved of observation in the construction of research theory, and finishing an analysis of emotional labour in the hospice setting. 


\subsection{Statistical Analysis of Data}

To establish its effectiveness after installation, EDMS was tested against five evaluation criteria created in accordance with the system's procedures and hypothesis formation:

- Information access is the benefit that will improve healthcare effectiveness by lowering the amount of time spent on administrative tasks.

- Clinical efficacy is described as the ability to support medical practitioners and patients by expediting clinical procedures and making information more accessible.

- Patient satisfaction is defined as the benefits perceived by the patient as a result of their complete treatment experience, from start to finish.

- Economic conditions are the need of investing in healthcare system reform

- Needs such as analyzing how the system functions, the convenience of use, addressing an industry need, adaptability, and time was met.

\section{Results and Discussion}

Two options were analyzed using the criteria as a guide, and the results are shown in the table below: The three-score levels are low, medium, and high. Low represents dissatisfaction, whereas High represents highly satisfied.

\begin{tabular}{|c|c|c|c|c|c|}
\hline Option & $\begin{array}{c}\text { Information } \\
\text { accessibility }\end{array}$ & Clinical efficacy & $\begin{array}{c}\text { Patient } \\
\text { satisfaction }\end{array}$ & $\begin{array}{c}\text { Econ- } \\
\text { situation } \\
\text { requirements } \\
\text { met }\end{array}$ \\
\hline Paper-based & Medium & Medium & Medium & Medium & High \\
\hline Paperless & High & High & High & Medium \\
\hline
\end{tabular}

Table 1. Evaluation Between Paper-Based and Paperless System

According to the results of the examination in Table 1, the paperless option has the potential to give greater benefits since it involves the full digitization of medical data. However, it is more difficult to adapt due to the more intricate capabilities and broader organizational footprint required.

Because of the paperless option, employees may track a medical record in real-time and know where it is at all times. This technique has the potential to deliver further benefits, but it will limit the number of suppliers accessible and is mostly unproven in the healthcare market. On a technological level, this feature is currently being studied.

\section{Conclusion and Recommendation}

According to our observations and significant research, the following are the key risks associated with sourcing an EDMS in the Philippines:

- Lack of competition in the digital infrastructure market and investment limitations in the telecommunications industry is harming attempts to strengthen the country's digital infrastructure. The Philippines continues to have slow internet connections, making it hard for EDMS to be successful in its present condition.

- Finances and the economy. The Philippines is now dealing with a shortage of funding for healthcare and telecommunications, all of which are still being handled. If the healthcare industry agrees to invest in EDMS, the next difficulty will be assuring the system's long-term survival.

As the relevance of digitalization grows, the role of electronic medical records systems (EDMS) becomes more significant since it corresponds to the quality and systematic approach in healthcare operations. If an EDMS is not fully implemented in the future, the likely impact will be the removal of the positive impact on patient care and safety that an EDMS will produce; the negative impact on medical practitioners, despite the fact that it has been demonstrated that the implementation of an EDMS would positively impact appointment times and allow medical practitioners to access information quickly and securely; and the cost of paper, space, and loss of medical data. 
An EDMS provider must extensively check and analyze the workflow to establish the system's competency and desire to meet the criteria of the healthcare business. More significantly, the Philippine healthcare sector should set aside a particular budget to invest in this sort of system if they want to increase the quality of patient care while simultaneously improving Filipinos' life expectancy.

\section{References}

[1] Afonso, C, Schwarz, A, Roldán, J \& Manuel J (2015). EDMS Use in Local E-Government: An Analysis of the Path from Extent of Use to Overall Performance. International Journal of Electronic Government Research. 11. 18-34.

[2] Ames, H., Glenton, C. \& Lewin, S (2019). Purposive sampling in a qualitative evidence synthesis: a worked example from a synthesis on parental perceptions of vaccination communication. BMC Med Res Methodol 19, 26.

[3] Berg M (2001). Implementing Information Systems in Healthcare Organizations: Myths and Challenges. International Journal of Medical Informatics; 64: 143-156.

[4] Bhattacherjee, A (2012). Social Science Research: Principles, Methods, and Practices. The University of South Florida. Textbooks Collection. 3.

[5] Blum B J, Lenhard Jr R E, Braine H, Kammer A (1977). A Clinical Information Display System. Annual Symposium on Computer Application in Medical Care: Washington, D.C., USA: 1977, 131-138

[6] Chee S, Ramaiah, C \& Prakash, G. (2010). Impact of electronic records on the healthcare industry. International Journal of Medical Toxicology and Legal Medicine. 13. 50-60.

[7] Coiera E (2003). Guide to Health Informatics. London: Oxford University Press.

[8] Green, C. \& Harding, T. (2013). Outline Business Case for a Trust-wide Electronic Document Management System. Sheffield Teaching Hospitals, NHS Foundation Trust. V.0.2, Issue Date: 06.11.2013

[9] Greenhalgh T, Russell J (2010). Why Do Evaluations of eHealth Programs Fail? An Alternative Set of Guiding Principles. PLoS Med ; 7 : (11) e1000360.

[10] Hamed T (2016). Sampling Methods in Research Methodology; How to Choose a Sampling Technique for Research. International Journal of Academic Research in Management (IJARM).

[11] Hirschheim R, Newman B (1991). Symbolism and Information Systems Development: Myth, Metaphor and Magic. Information Systems Research ; 2 : (1) 29-62.

[12] Institute of Medicine. (1997). The Computer-Based Patient Record: An Essential Technology for Health Care, Revised Edition. Washington, DC: The National Academies

[13] ISO/DTR 20154 (2004), Health Informatics - Electronic Health Record - Definition, Scope, and Context.

[14] Kabir, S. M. (2016). Methods Of Data Collection.

[15] Kainos Software (2010). Electronic Document Management in Healthcare - White Paper.

[16] Katani, M. (2014). Challenges of Implementing an Electronic Document Management System in a Large Health Care Facility in Southern California. The University of Victoria - School of Health Information Science.

[17] Kerkoulas P. S. (2002). How To Produce EDMS Requirements and Cost-Benefit Data. Topics in Health Information Management, 23:(1) 7-15.

[18] Rosa, A.T.R., Pustokhina, I.V., Lydia, E.L., Shankar, K., \& Huda, M. (2019). Concept of Electronic Document Management System (EDMS) as an Efficient Tool for Storing Document. Universitas Islam Nusantara, Bandung, Indonesia, Plekhanov Russian University of Economics, Moscow, Russia, Professor, Vignan's Institute of Information Technology(A), Department of Computer Science and Engineering, Visakhapatnam, Andhra Pradesh, India, Department of Computer Applications, Alagappa University, India, \& Universiti Pendidikan Sultan Idris, Malaysia. Vol 6, Issue 5, 2019

[19] Scott P J, Briggs J S (2010). STAT-HI: A Socio-Technical Assessment Tool for Health Informatics Implementations. The Open Medical Informatics Journal; 4: 214-220.

[20] Walshe, C., Ewing, G., \& Griffiths, J. (2011). Using observation as a data collection method to help understand patient and professional roles and actions in palliative care settings. Palliative Medicine, 26(8), 1048-1054. 